\title{
Treatment of osteoporosis with annual iv zoledronic acid: effects on hip fracture
}

\author{
Roland D Chapurlat \\ INSERM U83I, Université de Lyon, \\ Division of Rheumatology, Hôpital \\ E Herriot, Hospices Civils de Lyon, \\ Lyon, France
}

\begin{abstract}
Background: Several treatments for postmenopausal osteoporosis have been available in the past decade, but adherence to these treatments has been judged inadequate. The prevention of hip fracture by these medications is still modest.

Methods: A literature search was performed for treatment with zoledronic acid for the prevention of hip fracture.

Results: In the The Health Outcomes and Reduced Incidence with Zoledronic Acid Once Yearly (HORIZON) Pivotal Fracture Trial, involving 7765 postmenopausal women with low bone mineral density or with prevalent vertebral fracture, women taking zoledronic acid had a $41 \%$ relative risk reduction for hip fracture, at 3 years, compared to placebo. In the HORIZON Recurrent Fracture Trial, 2127 patients (76\% were women) were randomized to receive either zoledronic acid or placebo after sustaining a first hip fracture. A reduction of $30 \%$ in the second hip fracture risk was observed, but it did not reach statistical significance. Zoledronic acid was generally safe in these trials, although a slightly increased rate of severe atrial fibrillations was observed in the HORIZON Prevention Fracture Trial, but not in the HORIZON Recurrent Fracture Trial.

Conclusion: Yearly zoledronic acid reduces the risk of hip fracture, both in postmenopausal osteoporotic women with and without prevalent vertebral fracture and in men and women with a recent first hip fracture.
\end{abstract}

Keywords: osteoporosis, bisphosphonates, hip fracture, zoledronic acid

\section{Introduction}

Hip fracture is the most devastating complication due to bone fragility. Mortality is increased in women with hip fracture, $10 \%$ to $20 \%$ higher than expected for their age within the first year ${ }^{1,2}$ and this excess mortality persists for several years after hip fracture..$^{3,4}$ Loss of independence and entry in nursing homes are also common consequences of hip fractures. ${ }^{5}$ Prevention of hip fracture, however, is challenging because one needs to target both the bone - with anti-osteoporosis agents - and the triggering event that is generally a fall from standing height. In addition, hip fracture occurs mostly in individual over 75 years of age ${ }^{6}$ and the mean age at hip fracture is currently increasing. ${ }^{7,8}$ The second hip fracture is not a rare event, with an estimated incidence of around $2 \%$ per year. ${ }^{9,10}$ Elderly patients with hip fracture often present with several comorbidities hampering pharmacologic treatment because of drug interaction, and a negative influence on drug persistence. Cognitive impairment may also have a negative impact on drug persistence.

Preventing falls has proven to be difficult, although some studies suggest that vitamin D supplements, ${ }^{11}$ certain types of exercise, including tai chi, ${ }^{12}$ and cataract surgery $^{13}$ might reduce the risk of fall. Limiting the consequences of the fall may be possible with hip protectors, but their effectiveness has never been convincingly established, mainly due to poor compliance. ${ }^{14}$

A reduction in the incidence of hip fracture has been observed in some trials testing the efficacy of oral alendronate and risedronate, ${ }^{15-17}$ but the magnitude of fracture 
risk reduction was about half of that observed for vertebral fracture efficacy. For the other drugs the evidence is less compelling. In the BONE trial, examining the effect of ibandronate on fracture risk, no efficacy on hip fracture events could be obtained in the primary intent to treat analysis. ${ }^{18}$ With teriparatide, a 50\% reduction in non-vertebral fracture risk was shown, but the hip fracture risk reduction was not significant. This latter result may stem from the study design, as women were too young to have a sizeable hip fracture incidence. ${ }^{19}$ With intact parathyroid hormone, no efficacy on non-vertebral fracture, and hip fracture specifically, was observed either. ${ }^{20}$ Although treatment with strontium ranelate has been able to diminish the rate of non-vertebral fracture by $16 \%$, it is only in a post-hoc analysis that a $33 \%$ reduction of hip fracture risk could be found in a sub-group of women at higher risk of hip fracture. ${ }^{21}$ No reduction in hip fracture risk and all non-vertebral fracture risk has been shown with the first selective estrogen receptor modulator (SERM) to be marketed for the treatment of postmenopausal osteoporosis, raloxifene. ${ }^{22}$ With a new, more potent compound, lazofoxifene, it has been shown that a reduction of $24 \%$ in the incidence of non-vertebral fracture risk could be obtained, but the $23 \%$ reduced risk in hip fracture risk did not reach statistical significance. ${ }^{23}$

So, there is a clear need for new drugs to improve reduction in hip fracture risk. These drugs should be adequately tolerated in the elderly and their regime should be acceptable in those patients who often have many co-prescriptions. In this context, we will review the effectiveness of the treatment with annual intravenous zoledronic acid to prevent hip fracture.

\section{Methods}

Our literature search in PubMed used the following terms: zoledronic acid and hip fracture (50 articles), bisphosphonates and hip fracture (693 articles). Titles and/or abstracts were reviewed to determine the relevance for this review. We have selected articles relevant to the prevention of hip fracture with zoledronic acid, including all clinical trials and some relevant papers on the pharmacology of the compound and some studies related to the clinical use of bisphosphonates. We have also used studies on zoledronic acid we were aware of, which had been released only in the form of abstracts at the time of preparation of this article.

\section{Pharmacology of zoledronic acid}

Zoledronic acid is a potent nitrogen-containing BP, which has been widely used in the treatment of Paget's disease of bone, hypercalcemia, multiple myeloma, androgen-def iciency-induced bone loss in prostate cancer patients, prostate cancer bone metastases, and osteolytic bone metastases. This BP has also been marketed more recently for the treatment of postmenopausal osteoporosis, and should be available soon for the treatment of male osteoporosis and of glucocorticoid-induced bone loss.

In rat models, zoledronic acid - administered with subcutaneous injections for 10 days - produces dose-dependent increases in cancellous bone volume and connectivity, 100 -times more effectively than pamidronate, and decreases bone resorption. ${ }^{24}$

In another animal model of postmenopausal bone loss - the ovariectomized adult rhesus monkey - animals were randomly assigned to one control group and four ovariectomy groups. The control and one ovariectomized group received saline, and the three other ovariectomized groups were given 0.5, 2.5 or $12.5 \mu \mathrm{g} / \mathrm{kg}$ by a single weekly subcutaneous injection, for 69 weeks. ${ }^{25}$ Spine, total body and radius bone mineral density (BMD) were either preserved or increased by zoledronic acid, dose-dependently, up to week 39, and stabilized thereafter. Simultaneously, biochemical markers of bone turnover decreased significantly in monkeys receiving zoledronic acid, proportionally to the dosage, and remained reduced until the end of the study, compared with animals on placebo.

In a phase II, randomized, double-blind, placebo-controlled trial, zoledronic acid or placebo were given intravenously to 351 postmenopausal women with low BMD (T score $\leq 2$ ), for 1 year. These patients received placebo or intravenous zoledronic acid at doses of $0.25,0.5$ or $1.0 \mathrm{mg}$ every 3 months, or $2.0 \mathrm{mg}$ every 6 months, or a single annual $4.0 \mathrm{mg}$ dose. ${ }^{26}$ All women received a $1 \mathrm{~g}$ per day calcium supplement. All zoledronic acid regimens produced similar increases in lumbar spine and femoral neck BMD, spanning $4.3 \%$ to $5.1 \%$ at the spine, compared with stable BMD in the placebo group. Biochemical markers of bone resorption (urinary $\mathrm{N}$-terminal telopeptide of type I collagen [NTX], serum C-terminal telopeptide of type I collagen [CTX]) were suppressed during the trial, while markers of bone formation were also diminished, albeit later after beginning treatment. Post-infusion myalgia and transient fever were the most common adverse events.

Two consecutive, open-label extensions of this phase II study have been conducted over 5 years. ${ }^{27}$ In the first extension, most patients received $4 \mathrm{mg}$ once a year. They entered the second extension thereafter and received either zoledronic acid $4 \mathrm{mg}$ per year or calcium alone. Patients were analyzed according to the duration of their treatment with zoledronic acid (2, 3 or 5 years). Substantial increases in BMD were observed in all groups, along with significant reduction in 
biochemical markers of bone turnover. A third of patients, however, did not benefit from an optimal reduction in bone-specific alkaline phosphatase, even after several zoledronic acid infusions. This frequent suboptimal response was not observed when examining results of serum CTX-1 measurements. Taken together, these results may suggest that a $4 \mathrm{mg}$ yearly infusion may not have been adequate for all these postmenopausal patients.

The one-year phase II trial26 has paved the way for conducting a large phase III trial program (the HORIZON trials) designed to demonstrate the ability of zoledronic acid $5 \mathrm{mg}$ once a year given intravenously to reduce osteoporotic fracture risk.

\section{Clinical results with zoledronic acid in the prevention of hip fracture}

Results of two large phase III multicenter randomized placebo-controlled trials have been published so far. The HORIZON Pivotal Fracture Trial has been conducted among postmenopausal osteoporotic women with and without prevalent fracture. ${ }^{27}$ The HORIZON Recurrent Fracture Trial has tested the ability of the compound to reduce fracture risk among men and women with a recent hip fracture. ${ }^{28}$

\section{The HORIZON Pivotal Fracture Trial}

The Health Outcomes and Reduced Incidence with Zoledronic Acid Once Yearly (HORIZON) Pivotal Fracture Trial was an international, multicenter, randomized, doubleblind, placebo-controlled trial in postmenopausal women with osteoporosis, who were randomly assigned to receive either a 15-minute intravenous injection of zoledronic acid (5 mg) or placebo, at baseline (day 0), at 12 months, and at 24 months. All women received oral daily calcium (1000 to $1500 \mathrm{mg}$ ) and vitamin D (400 to $1200 \mathrm{IU}$ ) and were monitored for 3 years, to estimate the incidence of new vertebral and non-vertebral fracture.

Postmenopausal women between the ages of 65 and 89 years could be enrolled if they had a BMD T-score of -2.5 or less at the femoral neck, with or without prevalent vertebral fracture, or a T-score of -1.5 or less, with radiological evidence of at least two mild vertebral fractures or one moderate vertebral fracture. Previous use of oral bisphosphonates was permitted, the duration of the washout period depending on the duration of previous use. Concomitant use of several other antiosteoporosis drugs was allowed, such as raloxifene, calcitonin, tibolone, tamoxifen, dehydroepiandrosterone, ipriflavone, and medroxyprogesterone. Patients were stratified according to these baseline osteoporosis therapies. Patients with no bone medication at baseline were in stratum 1 , whereas those in stratum 2 were on an antiosteoporosis drug when they started the trial.

Two primary endpoints were adopted in this trial: the incidence of new vertebral fracture in stratum 1 and the incidence of hip fracture in both strata 1 and 2. Secondary efficacy endpoints included any non-vertebral fracture, any clinical fracture, and clinical vertebral fracture. Other secondary endpoints were changes in BMD at the total hip, femoral neck, and lumbar spine, and changes in markers of bone resorption (serum C-telopeptide of type I collagen, CTX) and formation (bone-specific alkaline phosphatase, BSAP, and N-terminal propeptide of type I collagen, PINP).

A total of 7765 women were randomized to receive either zoledronic acid $(n=3889)$ or placebo $(n=3876)$. Their mean age was 73 years, 63\% had prevalent vertebral fracture and $79 \%$ had no other osteoporosis medication at baseline (stratum 1). Eighty-four percent of patients were still followed-up after 3 years.

The relative risk reduction for hip fracture in women taking zoledronic acid was $41 \%$ with zoledronic acid and the vertebral fracture relative risk reduction at 3 years was $70 \%$. Fracture risk reduction after 1 and 2 years of treatment was comparable to that observed after 3 years. Non-vertebral fractures were reduced by $25 \%$, all clinical fractures by $33 \%$ and clinical vertebral by $77 \%$, in women on zoledronic acid.

BMD at the hip increased by $6 \%$ and at the spine by $6.7 \%$ after 3 years of follow-up, compared with the placebo. All three markers of bone turnover (serum CTX, BSAP and PINP) decreased significantly compared with the placebo group, when they were measured 12 months after starting therapy. At 6 and 12 months after each infusion, there was no further decline in their concentrations.

The numbers of patients who died, had a serious adverse event, or discontinued follow-up because of an adverse event did not significantly differ between the study groups. Among patients on zoledronic acid, 31.6\% had a brief flu-like syndrome after the first infusion, compared with $6.2 \%$ in the placebo group. A transient increase of more than $0.5 \mathrm{mg} / \mathrm{dL}$ in serum creatinine levels was observed 9 to 11 days after the infusion, among $1.3 \%$ of patients with zoledronic acid and $0.5 \%$ of patients in the placebo group. The number of patients who had arrhythmia in the zoledronic acid group (266 patients, or $6.9 \%$ ) was significantly higher than that in the placebo group (203 patients, or $5.3 \% ; \mathrm{p}=0.003$ ), as well as serious atrial fibrillation. In the zoledronic acid group, 50 patients had serious atrial fibrillation (1.3\%), compared with 20 patients $(0.5 \%)$ in the placebo group. Among these 
50 serious atrial fibrillation events, 47 occurred more than 30 days after the infusion. The occurrence of stroke, or death from stroke, did not differ between the two groups. Two cases of potential osteonecrosis of the jaw were identified (one in the placebo group and one in the zoledronic acid group).

In a substudy of the HORIZON fracture trial, 152 patients underwent a bone biopsy. Zoledronic acid reduced bone turnover by a median $63 \%$ and preserved bone structure and volume, with evidence of ongoing bone remodeling in $99 \%$ of the biopsies. ${ }^{30}$ Bone formation was normal, with no evidence of mineralization defect. The concomitant administration of other antiosteoporosis drugs did not affect the tissue-level response to zoledronic acid.

\section{The HORIZON Recurrent Fracture Trial}

The Health Outcomes and Reduced Incidence with Zoledronic Acid Once Yearly (HORIZON) Recurrent Fracture Trial was an international, multicenter, randomized, placebocontrolled trial involving men and women with recent hip fracture. ${ }^{29}$ Patients were randomized to receive a yearly infusion of either $5 \mathrm{mg}$ of zoledronic acid or an infusion of placebo. The treatment was administered within 90 days of the surgical repair of the hip fracture, and once a year thereafter. All patients were supplemented with calcium and a loading dose of vitamin D was administered soon after the first hip fracture. All these patients were unable or unwilling to take an oral bisphosphonate. A parallel therapy with calcitonin, a SERM, tibolone and hip protectors was authorized.

The primary endpoint was a new clinical fracture. Secondary endpoints included a second hip fracture and death, as well as the change in BMD at the non-fractured hip, and new vertebral and non-vertebral fracture. Among 2127 patients, 1065 received zoledronic acid and 1062 were on placebo, and $76 \%$ were women.

After a median follow-up of 1.9 years, the observed fracture rate of clinical fracture was $8.6 \%$ in the zoledronic acid group and $13.9 \%$ in the placebo group, representing an absolute risk reduction of $5.3 \%$ and a relative risk reduction of $35 \%$. There was also a non-significant $30 \%$ second hip fracture relative risk reduction associated with the use of zoledronic acid. Death from all causes was reduced by $28 \%$ in the zoledronic acid group. BMD at the total hip increased in the zoledronic acid group by $2.6 \%$ at 12 months, $4.7 \%$ at 24 months, and 5.5\% at 36 months and declined in the placebo group by $1.0 \%, 0.7 \%$, and $0.9 \%$, respectively.

The incidence of pyrexia, myalgia and bone pain was increased among patients who received zoledronic acid, whereas the incidence of serious adverse events was comparable in the groups. Similarly, there was no difference for the occurrence of serious renal adverse events, arrhythmia and atrial fibrillation. No case of osteonecrosis of the jaw was reported.

\section{Discussion}

Among postmenopausal women with osteoporosis, zoledronic acid administered once a year at $5 \mathrm{mg}$ was associated with a $40 \%$ reduction in hip fracture risk. In men and women receiving the same regimen starting shortly after a first hip fracture, a trend toward a one third reduction in second hip fracture incidence has been observed.

Although no direct comparison in head-to-head trials examining fracture efficacy has been made in comparison with other antiresorptive drugs, prescribed orally, the magnitude of the effect looks somewhat larger with zoledronic acid. It is also possible to make an indirect comparison between placebo-controlled trials, as outlined by Bucher and $\mathrm{al}^{31}$ In indirect comparisons examining risedronate vs zoledronic acid and alendronate vs zoledronic acid, there was no significant difference between these drugs for hip fracture efficacy. ${ }^{32}$ Several assumptions, however, need to be made when those calculations are made. First, the samples of patients in the different trials must be drawn from the same kind of population. This is not necessarily the case in these studies, as inclusion/exclusion criteria differed markedly between these trials. Second, the relative risks have to be derived from the intent-to-treat analyses, which was the case. Third, the dropouts must be accounted for in the trials, which was possible. Rates of compliance, definition of outcomes and methodologic quality of the trials can also affect the inferences from these indirect comparisons. So, at this stage, there is no absolute answer as to whether the hip fracture risk reduction observed with zoledronic acid is more important than with other bisphosphonates.

The Pivotal Fracture Trial involved both women with and without prevalent fracture. This the first time that a reduction in hip fracture risk has been demonstrated in a population treated with bisphosphonates including women with no prevalent vertebral fracture. So, this compound appears to be efficacious at various stages of the severity of postmenopausal osteoporosis.

The Recurrent Fracture Trial is the first trial with an antiosteoporosis drug that showed a decrease in mortality. This reduction was observed after about 16 months of follow-up. Given the number of fractures that were avoided by the reduction of bone fragility, the reduction in fracture risk explained $2 \%$ of the mortality reduction. ${ }^{33}$ In a post-hoc sub-group 
analysis, men experienced a greater mortality benefit, with fewer cardiac-related events. Death from pneumonia, neoplasms, and cardiovascular disease in patients treated with zoledronic acid were reduced, whereas the incidence of these diseases was similar to that in the placebo group, suggesting that the drug might influence physiologic reserves.

One of the limitations of the first oral bisphosphonates was their daily dosage. Weekly regimens have been developed later to diminish the treatment annoyance. In a recent analysis, $46 \%$ of women on a weekly formulation were still taking their drug at 1 year, whereas only $33 \%$ of those on a daily dosage were still on medication. ${ }^{34}$ Another limitation of oral bisphosphonates is the possibility of digestive adverse events such as abdominal pain, dyspepsia and, rarely, oesophagitis. Persistence on antiosteoporosis drugs is poor, like with most chronic diseases. ${ }^{34}$ Thus, in a cohort of older american women, drug persistence was only $45 \%$ 1 year after starting a treatment for osteoporosis. ${ }^{35}$ In another analysis performed in California, only $25 \%$ of women were still taking their medication at 1 year. ${ }^{36}$ In another study conducted in North America, raloxifene and bisphosphonates were frequently stopped and the main reason for treatment interruption was the occurrence of adverse events. ${ }^{37}$ Antifracture benefit, however, improves as much as compliance increases. ${ }^{38}$ The efficacy of zoledronic acid on hip fracture may be related, at least in part, to its iv administration, allowing for a better delivery of the drug at the bone level, compared with oral agents.

This effect of yearly intravenous zoledronic acid on hip fracture may also be influenced by the quick onset of the antiresorptive action. In a randomized, double blind, doubledummy trial comparing the onset of action of zoledronic acid $5 \mathrm{mg}$ and alendronate $70 \mathrm{mg}$ in postmenopausal women with low BMD, ${ }^{39}$ the reduction in markers of bone resorption was significantly greater at week one in women on zoledronic acid than in those on alendronate. The difference between the two drugs waned over time, but a slight difference in favor of zoledronic acid remained after 12 weeks.

The quality of trials, ie, their validity, is critical for clinical practice and for health care systems who reimburse the drugs. The drop-out rate must be low so the intention to treat analysis remains reliable, along with sound randomization and statistical analysis. A wide spectrum of the disease must be enrolled to allow for good generalizability of trial results. In the HORIZON Pivotal Fracture trial, ${ }^{28} 84 \%$ of patients remained in active follow-up and $81 \%$ received all three infusions. For comparison with other major phase III osteoporosis trials, $88 \%$ of patients were still taking the drug after 3 years in the FIT I trial testing alendronate, ${ }^{15}$ complete follow-up data at 3 years were available for only $64 \%$ of the women in the HIP trial examining risedronate, ${ }^{16}$ or $64 \%$ to $68 \%$ (according to different treatment groups) completed the study in the 3-year BONE trial testing the efficacy of oral ibandronate. ${ }^{18}$ In the HORIZON Recurrent Fracture Trial. ${ }^{29}$ which was an event-driven trial, $71 \%$ of the patients completed the trial, but only 3\% were lost to follow-up. Results from the HORIZON program apply to a large range of severity of postmenopausal osteoporosis, because women could have osteoporosis with or without prevalent vertebral fracture, the ages ranging from 65 to 89 . They could have already received certain osteoporosis medications, and there were few exclusion criteria. Patients in the HORIZON Recurrent Fracture Trial had a recent hip fracture.

Patients at risk of hip fracture are generally elderly, over 75. So, the tolerability of drugs is essential in this frail population. In the HORIZON Pivotal Fracture Trial, 2.3\% of women had hypocalcemia, defined as serum calcium below $2.075 \mathrm{mmol} / \mathrm{L}, 9$ to 11 days after the infusion. These episodes were transient and asymptomatic in all cases. In the HORIZON Recurrent Fracture Trial, although patients were older and more frail, fewer episodes of hypocalcemia were observed $(0.3 \%)$, possibly due to the loading dose of vitamin D patients received before their first infusion. So, hypocalcemia appears to be a rare and subclinical event in women receiving zoledronic acid. In older patients, however, it is probably wise to measure serum dihydroxy-vitamin D and to give an adequate vitamin D supplement, including a loading dose if judged necessary, to avoid hypocalcemia as much as possible.

In the HORIZON Pivotal Fracture Trial, but not in the HORIZON Recurrent Fracture Trial, serious atrial fibrillation occurred more frequently in the zoledronic acid group $(1.3 \%)$ than in the placebo group $(0.5 \%, \mathrm{p}<0.001)$. This finding has prompted several groups to report data on atrial fibrillation from other bisphosphonates trials. In the FIT trials, a trend was observed for the occurrence of serious atrial fibrillation, with a relative hazard of $1.51(0.97,2.40) .{ }^{40}$ In the phase III trials of risedronate for osteoporosis, there was no difference in the incidence of atrial fibrillation, stroke, or death related to cardiovascular disease between the placebo and treated groups. ${ }^{41}$ In a population-based case control study in Denmark based on medical databases, involving 13,694 patient with atrial fibrillation/flutter and 68,470 controls, there was no association between use of oral bisphosphonate and the risk of atrial fibrillation/flutter. ${ }^{42}$ In this context of conflicting data on the influence of bisphosphonates on the risk of atrial fibrillation, 
it remains possible that the observation of increased risk in the HORIZON Pivotal Fracture Trial is a chance finding induced by multiple comparisons. The possibility that this arrhythmia might be related to subclinical hypocalcemia and the resulting secondary hyperparathyroidism, ${ }^{43}$ however, points to the need for monitoring this potential cardiovascular event when zoledronic is marketed worldwide.

One of the concerns with new antiresorptive agents is their bone safety, regarding the risk of mineralization defects and the risk of over-suppression of bone turnover. In the ancillary bone biopsy study of the HORIZON trial, there was no mineralization defect. ${ }^{30}$ All 152 but 1 biopsies showed evidence of tetracycline label, indicating preserved remodeling capacity. One year after treatment, before the next infusion, an increase in serum markers of bone resorption was visible, consistent with this preservation of bone remodeling capacity. It is likely that zoledronic acid may be started among patients who have already received other bisphosphonates. In a randomized double blind double dummy trial, conducted in postmenopausal women with low BMD who had been previously treated with alendronate for an average 4 years, patients received either yearly zoledronic acid $5 \mathrm{mg}$ or oral weekly alendronate $70 \mathrm{mg}$ over 1 year. ${ }^{44} \mathrm{BMD}$ was maintained in both groups, with no significant difference in BMD change between the two groups. In addition, zoledronic acid further diminished markers of bone resorption, which increased thereafter, suggesting a preserved bone remodeling capacity. Thus, there is ample evidence for satisfactory bone safety with the use of yearly zoledronic acid over 3 years.

Osteonecrosis of the jaw associated with the use of bisphosphonates has been described since 2003. It is defined by exposed bone in the maxillofacial region during at least 8 weeks, in patients previously or currently taking bisphosphonates, who did not receive radiotherapy of the maxillofacial region. ${ }^{45}$ The incidence of osteonecrosis of the jaw among patients receiving monthly infusions of pamidronate or zoledronic acid to treat malignancy ranges between $1 \%$ and $10 \%$. In patients taking bisphosphonates for the treatment of Paget's disease of bone or osteoporosis, it seems to be an exceptional event, with an incidence of $1 / 100,000$ per patient-years. In the HORIZON Pivotal Fracture Trial, there were one case in the placebo group and one case in the zoledronic acid group. No case of osteonecrosis of the jaw was identified in the HORIZON Recurrent Fracture Trial. Prior studies show that the incidence of osteonecrosis of the jaw is proportional to the cumulative dose of bisphosphonate, explaining the much higher incidence among patients with malignancies than those with osteoporosis. It is likely that the incidence of osteonecrosis of the jaw that might be observed in patients receiving zoledronic acid is comparable to that encountered with previous oral bisphosphonates used for osteoporosis. The occurrence of this adverse event is certainly related to the dose and not to the mode of administration. So, at this stage, it has been recommended by the American Society for Bone and Mineral Research Task Force ${ }^{45}$ that patients be informed about the risk of osteonecrosis of the jaw, maintain good oral hygiene and have regular dental visits. There is no contraindication to dental implant placement. Current evidence supports the same recommendations for annual zoledronic acid as for older oral bisphosphonate therapies.

\section{Conclusion}

Yearly intravenous zoledronic acid $5 \mathrm{mg}$ is an effective treatment to reduce the incidence of hip fracture among postmenopausal osteoporotic women. A trend toward a second hip fracture risk reduction has also been observed in patients with a first hip fracture. This treatment has been well tolerated in clinical trials.

\section{Disclosures}

The author discloses no conflicts of interest.

\section{References}

1. Center JR, Nguyen TV, Schneider D, Sambrook PN, Eisman JA. Mortality after all major types of osteoporotic fracture in men and women: an observational study. Lancet. 1999;353:878-882.

2. Cummings SR, Melton LJ. Epidemiology and outcomes of osteoporotic fractures. Lancet. 2002;359:1761-1767.

3. Trombetti A, Hermann F, Hoffmeyer P, Schurch MA, Bonjour JP, Rizzoli R. Survival and potential years of life lost after hip fracture in men and age-matched women. Osteoporos Int. 2002;13:731-737.

4. Farahmand BY, Michaelsson K, Ahlbom, A et al. Survival after hip fracture. Osteoporos Int. 2005;16:1583-1590.

5. Bonar SK, Tinetti ME, Speechley M, Cooney LM. Factors associated with short- versus long-term skilled nursing facility placement among community-living hip fracture patients. J Am Geriatr Soc. 1990;38:1139-1144.

6. Cummings SR, Nevitt MC, Browner WS, et al. Risk factors for hip fracture in white women. N Engl J Med. 1995;332:767-773.

7. Couris CM, Duclos A, et al. A seventy percent overestimation of the burden of hip fractures in women aged 85 and over. Bone. 2007; 41:896-900

8. Chevalley T, Guilley E, Hermann FR, Hoffmeyer P, Rapin CH, Rizzoli R. Incidence of hip fracture over a 10-year period (1991-2000): reversal of a secular trend. Bone. 2007;1284-1289.

9. Chapurlat RD, Bauer DC, Nevitt M, Stone K, Cummings SR. Incidence and risk factors for a second hip fracture in elderly women. The study of osteoporotic fractures. Osteoporos Int. 2003;14:130-136.

10. Berry SD, Samelson EJ, Hannan MT, et al. Second hip fracture in older men and women: the Framingham Study. Arch Int Med. 2007; 167:1971-1976.

11. Pfeifer M, Begerow B, Minne HW, Suppan K, Fahrleitner-Pammer A, Dobnig H. Effects of long-term vitamin D and calcium supplementation on falls and parameters of muscle function in community-dwelling older individuals. Osteoporos Int. 2009;20(2):315-322. 
12. Wolf SL, Barnhart HX, Kutner NJ, et al. Reducing frailty and falls in older persons: an investigation of tai chi and computerized balance training. J Am Geriatr Soc. 1996;44:489-497,

13. Harwood RH, Foss AJ, Osbom F, Gregson RM, Zaman A, Masud T. Falls and health status in elderly women following first eye cataract surgery:a randomised controlled trial. Br J Ophtalmol. 2005;89:53-59.

14. Kiel DP, Magaziner J, Zimmerman S, et al. efficacy of a hip protector to prevent hip fracture in nursing home residents: the HIP PRO randomized controlled trial. JAMA. 2007;298:413-422.

15. Black DM, Cummings SR, Karpf DB, et al. Randomised trial of effect of alendronate on risk of fracture in women with existing vertebral fractures. Lancet. 1996;348:1535-1541.

16. McClung MR, Geusens P, Miller PD, et al. Effect of risedronate on the risk of hip fracture in elderly women. $N$ Engl J Med. 2001;344: 333-340.

17. Boonen S, Laan RF, Barton IP, Watts NB. Effect of osteoporosis treatments on risk of non-vertebral fractures:review and meta-analysis of intention-to-treat studies. Osteoporos Int. 2005;16:1291-1298.

18. Chestnut CH III, Skag A, Christiansen C, et al. Effects of oral ibandronate administered daily or intermittently on fracture risk in postmenopausal osteoporosis. J Bone Miner Res. 2004;19:1241-1249.

19. Neer RM, Arnaud CD, Zanchetta JR, et al. Effect of parathyroid hormone (1-34) on fractures and bone mineral density in post-menopausal women with osteoporosis. N Engl J Med. 2001;344:1434-1441.

20. Greenspan SL, Bone HG, Ettinger MP, et al. Effect of recombinant human parathyroid hormone (1-84) on vertebral fracture and bone mineral density in postmenopausal women with osteoporosis:a randomized trial. Ann Intern Med. 2007;146:326-339.

21. Reginster JY, Seeman E, De Vernejoul MC, et al. Strontium ranelate reduces the risk of non vertebral fractures in postmenopausal women with osteoporosis:treatment of peripheral osteoporosis (TROPOS) study. J Clin Endocrinol Metab. 2005;90:2816-2822.

22. Ettinger B, Black DM, Mitlak BH, et al. Reduction of vertebral fracture risk in postmenopausal women with osteoporosis treated with raloxifene:results from a 3-year randomized clinical trial. Multiple Outcomes of Raloxifene Evaluation (MORE) investigators. JAMA. 1999;282:637-645.

23. Cummings SR, Eastell R, Ensrud K, et al. The effect of lasofoxifene on fractures and breast cancer:3-year results from the PEARL Trial. J Bone Miner Res. 2008;23 Suppl 1:S81.

24. Pataki A, Muller K, Green JR, et al. Effects of short-term treatment with the bisphosphonates zoledronate and pamidronate on rat bone: a comparative histomorphometric study on the cancellous bone before, during and after treatment. Anat Rec. 1997;249:458-468.

25. Binkley N, Kimmel D, Bruner J, et al. Zoledronate prevents the development of absolute osteopenia following ovariectomy in adult rhesus monkeys. J Bone Miner Res. 1998;13:1775-1782.

26. Reid IR, Brown JP, Burkhardt P, et al. Intravenous zoledronic acid in postmenopausal women with low bone mineral density. $N$ Engl J Med. 2002;346:653-661.
27. Devogelaer JP, Brown JP, Burckhardt P, et al. Zoledronic acid efficacy and safety over five years in postmenopausal women. Osteoporos Int. 2007; 18:1211-1218.

28. Black DM, Delmas PD, Eastell R, et al. One-yearly zoledronic acid for treatment of postmenopausal osteoporosis. $N$ Engl J Med. 2007;356:1809-1822.

29. Lyles KW, Colon-Emeric CS, Magaziner JS, et al. Zoledronic acid and clinical fractures and mortality after hip fractures. $N$ Engl J Med. 2007;357:1799-1809.

30. Recker R, Delmas PD, Halse J, et al. The effects of intravenous zoledronic acid once yearly on bone remodelling and bone structure. $J$ Bone Miner Res. 2008;23:6-16.

31. Bucher HC, Guyatt GH, Griffith LE, Walter SD. The results of direct and indirect treatment comparisons in meta-analysis of randomized controlled trials J Clin Epidemiol. 1997;50:683-691.

32. Hochberg MC. Non vertebral fracture risk reduction with nitrogencontaining bisphosphonates Curr Osteopor Rep. 2008;6:89-94.

33. Colon-Emeric C, Mesenbrink P, Lyles K, et al. Potential mediators of the reduction in mortality with zoledronic avid after hip fracture. $J$ Bone Miner Res. 2008;23 Suppl 1:S10.

34. Cro JJ, Ishak KJ, Huybrechts KF, et al. The impact of compliance with osteoporosis therapy on fracture rates in actual practice. Osteoporos Int. 2004;15:1003-1008.

35. Solomon DH, Avorn J, Katz JN, et al. Compliance with osteoporosis medications. Arch Int Med. 2005;165:2414-2419.

36. McCombs JS. Compliance with drug therapies for the treatment and prevention of osteoporosis. Maturitas. 2004;48:271-287.

37. Tosteson A, Grove MR, Hammond, CS, et al. Early discontinuation of treatment for osteoporosis. Am J Med. 2003;115:209-216.

38. Caro JJ, Ishak KJ, Huybrechts KF, Raggio G, Naujoks C. The impact of compliance with osteoporosis therapy on fractures rates in actual practice. Osteoporos Int. 2004;15:1003-1008.

39. Saag K, Lindsay R, Kriegman A, et al. A single zoledronic acid infusion reduces bone resorption markers more rapidly than weekly oral alendronate in postmenopausal women with low bone mineral density. Bone. 2007;40:1238-1243.

40. Cummings SR, Schwartz AV, Black DM. Alendronate and atrial fibrillation. $N$ Engl J Med. 2007;356:1895-1896.

41. Karam R, Camm J, McClung M. Yearly zoledronic acid in postmenopausal osteoporosis. N Engl J Med. 2007;357(7):712-713.

42. Sorensen HT, Christensen S, Mehnert F, et al. Use of bisphosphonates and risk of atrial fibrillation and flutter. $B M J .2008 ; 336(7648)$ : 813-816.

43. Poole KES, Kaptoge S, Reeve J. Yearly zoledronic acid in postmenopausal osteoporosis. N Engl J Med. 2007;357:711-712.

44. McClung M, Recker R, Miller P, et al. Intravenous zoledronic acid $5 \mathrm{mg}$ in the treatment of postmenopausal women with low bone density previously treated with alendronate. Bone. 2007;41:122-128.

45. Khosla S, Burr D, Cauley J, et al. Bisphosphonate-associated osteonecrosis of the jaw: report of a task force of the American Society for Bone and Mineral Research. J Bone Miner Res. 2007;22:1479-1491. 
